\title{
A Truncation Method for Solving the Time-Fractional Benjamin-Ono Equation
}

\author{
Mohamed R. Ali (iD \\ Department of Mathematics, Benha Faculty of Engineering, Benha University, Benha, Egypt \\ Correspondence should be addressed to Mohamed R. Ali; mohamed.reda@bhit.bu.edu.eg \\ Received 12 December 2018; Revised 2 March 2019; Accepted 25 March 2019; Published 2 May 2019 \\ Academic Editor: Mustafa Inc \\ Copyright ( 2019 Mohamed R. Ali. This is an open access article distributed under the Creative Commons Attribution License, \\ which permits unrestricted use, distribution, and reproduction in any medium, provided the original work is properly cited. \\ We deem the time-fractional Benjamin-Ono (BO) equation out of the Riemann-Liouville (RL) derivative by applying the Lie \\ symmetry analysis (LSA). By first using prolongation theorem to investigate its similarity vectors and then using these generators to \\ transform the time-fractional BO equation to a nonlinear ordinary differential equation (NLODE) of fractional order, we complete \\ the solutions by utilizing the power series method (PSM).
}

\section{Introduction}

Lie symmetry method provides an effective tool for deriving the analytic solutions of the nonlinear partial differential equations (NLPDEs) [1-4]. In recent years, many authors have studied the nonlinear fractional differential equations (NLFDEs) because these equations express many nonlinear physical phenomena and dynamic forms in physics, electrochemistry, and viscoelasticity [5-9].

Time-fractional NLDEs arise from classical NLPDEs by replacing its time derivative with the fractional derivative. The methods applied to derive the analytic solutions of NLFPDEs are the exp-function, the $G^{\prime} / G$ expansion, fractional su-equation, Lie symmetry method, and many more [10-19].

The one-dimensional Benjamin-Ono equation is considered here as follows (see [20]):

$$
u_{t}+h u_{x x}+u u_{x}=0
$$

In fact, the $\mathrm{BO}$ equation describes one-dimensional internal waves in deep water. We consider LSA for the analytic solutions by using PS expansion for the time-fractional $\mathrm{BO}$ equation:

$$
u_{t}^{\alpha}+h u_{x x}+u u_{x}=0, \quad 0<\alpha<1
$$

In division 2 of this paper, some basic properties of the Riemann-Liouville fractional derivative are shown firstly and then the Lie group method for FPDEs is presented. In division 3 , the Lie group to the time-fractional $\mathrm{BO}$ equation (FBO) and the symmetry reductions are determined. In division 4, we derive anew arrangement of the FBO equation (2) via the PSM. In division 5, we study the convergence for the series solution. We conclude our work in division 6.

\section{Notations and Delineations}

2.1. Description of Lie Symmetry Reduction Method for NLFPDEs. We present the principal notations and definitions that detecting the symmetries of the NLFPDEs.

Here, the time-fractional NLFPDEs are

$$
\partial_{t}^{\alpha} u=F\left(t, x, u, u_{x}, u_{x x}, \ldots \ldots\right)
$$

Suppose that the infinitesimal vector $X$ has the form

$$
X=\xi^{1}(x, t, u) \frac{\partial}{\partial x}+\xi^{2}(x, t, u) \frac{\partial}{\partial t}+\eta(x, t, u) \frac{\partial}{\partial u}
$$

The Lie group parameter of infinitesimal transformations [8, $21,22]$ has the formula 


$$
\begin{aligned}
\bar{x} & =x+\varepsilon \xi^{1}(t, x, u)+O\left(\varepsilon^{2}\right), \\
\bar{t} & =t+\varepsilon \xi^{2}(t, x, u)+O\left(\varepsilon^{2}\right), \\
\bar{u} & =u+\varepsilon \eta(t, x, u)+O\left(\varepsilon^{2}\right), \\
\frac{\partial^{\alpha} \bar{u}}{\partial \bar{t}^{\alpha}} & =\frac{\partial^{\alpha} u}{\partial t^{\alpha}}+\varepsilon \eta_{\alpha}^{0}(t, x, u)+O\left(\varepsilon^{2}\right), \\
\frac{\partial \bar{u}}{\partial \bar{x}} & =\frac{\partial u}{\partial x}+\varepsilon \eta^{x}(t, x, u)+O\left(\varepsilon^{2}\right), \\
\frac{\partial^{2} \bar{u}}{\partial \bar{x}^{2}} & =\frac{\partial^{2} u}{\partial x^{2}}+\varepsilon \eta^{x x}(t, x, u)+O\left(\varepsilon^{2}\right),
\end{aligned}
$$

where $\xi^{1}, \xi^{2}$, and $\eta$ are considered as the infinitesimals of the transformation's variables $(x, t, u)$, respectively, and $\varepsilon$ ń1 is considered as the group parameter; we will take it to be equal to one. The explicit expressions of $\eta^{x}$ and $\eta^{x x}$, which we consider as the prolongation of the infinitesimals, are given by

$$
\eta^{x}=D_{x}(\eta)-u_{x} D_{x}\left(\xi^{1}\right)-u_{t} D_{t}\left(\xi^{2}\right)
$$

and

$$
\eta^{x x}=D_{x}\left(\eta^{x}\right)-u_{x t} D_{x}\left(\xi^{1}\right)-u_{x x} D_{t}\left(\xi^{2}\right)
$$

where $D_{x}$ is in [8] assigned as

$$
D_{x}=\frac{\partial}{\partial x}+u_{x} \frac{\partial}{\partial x}+u_{x x} \frac{\partial}{\partial u_{x}}+\ldots
$$

Theorem 1. Equation (2) coincides with a one-parameter group of transformations (5) with the infinitesimal generator $X$ if and only if the accompanying infinitesimal conditions holds true:

$$
\left.\operatorname{Pr}^{(\alpha, 2)} X(\Delta)\right|_{\Delta=0}=0
$$

where $\Delta=D_{t}^{\alpha} u-F\left(t, x, u, u_{x}, u_{x x}, \ldots ..\right)$ and $\operatorname{Pr}$ is the second prolongation of the infinitesimal generator $X$.

Definition 2. The prolonged vector is demonstrated by

$$
\begin{aligned}
\operatorname{Pr}^{(n)} X= & X+\sum_{i=1}^{p} \sum_{\alpha=1}^{q} \xi_{i}^{\alpha} \frac{\partial}{\partial u_{i}^{\alpha}}+\ldots \\
& +\sum_{j_{1=1}}^{p} \ldots \sum_{j_{n}=1}^{p} \sum_{\alpha=1}^{q} \xi_{j_{1} \ldots j_{n}}^{\alpha} \frac{\partial}{\partial u_{j_{1} \ldots j_{n}}^{\alpha}}
\end{aligned}
$$

where $q$ is the number of dependent variables, $p$ is the number of independent variables, $\partial / \partial u_{j_{1}}^{\alpha}=\partial / \partial u_{x}^{\alpha}$, and the PDE involves derivatives of up to the order $n$. The condition [21-23] is given by

$$
\left.\xi^{2}(t, x, u)\right|_{t=0}=0
$$

Lemma 3. The function $u=\theta(x, t)$ is an invariant solution of (3) if and only if

$$
\text { (i) } \xi^{2}(x, t, \theta) \theta_{t}+\xi^{1}(x, t, \theta) \theta_{x}=\eta(x, t, \theta) \text {. }
$$

Lemma 4. The $\boldsymbol{\alpha}^{\text {th }}$ extended infinitesimal [24, 25] for the fractional derivative part utilizing the $R L$ definition with (11) is given by

$$
\begin{aligned}
\eta_{\alpha}^{0} & =\frac{\partial^{\alpha} \eta}{\partial t^{\alpha}}+\left(\eta_{u}-\alpha D_{t}\left(\xi^{2}\right)\right) \frac{\partial^{\alpha} u}{\partial t^{\alpha}}-u \frac{\partial^{\alpha} \eta_{u}}{\partial t^{\alpha}}+\mu \\
& -\sum_{n=1}^{\infty}\left(\begin{array}{l}
\alpha \\
n
\end{array}\right) D_{t}^{n}\left(\xi^{1}\right) D_{t}^{\alpha-n}\left(u_{x}\right)+\sum_{n=1}^{\infty}\left[\left(\begin{array}{l}
\alpha \\
n
\end{array}\right) \frac{\partial^{\alpha} \eta_{u}}{\partial t^{\alpha}}\right. \\
& -\left[\left(\begin{array}{c}
\alpha \\
n+1
\end{array}\right) D_{t}^{n+1}\left(\xi^{2}\right)\right] D_{t}^{\alpha-n}(u),
\end{aligned}
$$

where

$$
\mu=\sum_{n=2}^{\infty} \sum_{m=2}^{n} \sum_{k=2}^{m} \sum_{r=2}^{k-1}\left(\begin{array}{l}
\alpha \\
n
\end{array}\right)\left(\begin{array}{l}
n \\
m
\end{array}\right)\left(\begin{array}{l}
k \\
r
\end{array}\right) \frac{1}{k !}
$$

Remember that

$$
\left(\begin{array}{l}
\alpha \\
n
\end{array}\right)=\frac{(-1)^{n-1} \alpha \Gamma(n-\alpha)}{\Gamma(1-\alpha) \Gamma(n+1)}
$$

\section{Reduction of Time-Fractional Benjamin-Ono Equation}

We use the LSA to find the similarity solution for 1D timefactional BO equation (1). Suppose that (2) is an invariant under (5), so that we have

$$
\bar{u}_{t}^{\alpha}+h \bar{u}_{x x}+\bar{u} \bar{u}_{x}=0
$$

Thus, $u(x, t)$ satisfies (2). Applying the second prolongation to (2), symmetry invariant equation is

$$
\eta_{\alpha}^{0}+u \eta^{x}+h \eta^{x x}+u_{x} \eta=0
$$

Substituting the values from (6), (7), and (12) into (16) and isolating coefficients in partial derivatives regarding $x$ and power of $u$, we have

$$
\begin{aligned}
& \left(\begin{array}{l}
\alpha \\
n
\end{array}\right) \partial_{t}^{n} \eta_{u}-\left(\begin{array}{c}
\alpha \\
n+1
\end{array}\right) D_{t}^{n+1}\left(\xi^{2}\right)=0, \quad n=1,2,3 \ldots \\
& \xi_{u}{ }^{2}=\xi_{x}{ }^{2}=\xi_{u}{ }^{1}=\xi_{t}{ }^{1}=\eta_{u u}=0,
\end{aligned}
$$




$$
\begin{aligned}
& \alpha \xi_{t}^{2}-2 \xi_{x}^{1}=0, \\
& h \eta_{x x}-u \partial_{t}^{\alpha} \eta_{u}+\partial_{t}^{\alpha} \eta+u \eta_{x}=0, \\
& \xi_{x x}{ }^{1}-2 \eta_{x u}=0 .
\end{aligned}
$$

Solving the obtained determining equation, we get

$$
\begin{aligned}
& \xi^{1}=c_{2}+\alpha x c_{1}, \\
& \xi^{2}=2 t c_{1}, \\
& \eta=-\alpha u c_{1},
\end{aligned}
$$

where $c_{1}$ and $c_{2}$ are constants, for simplicity. We take their values equal to one. So, (2) has two vector fields that can generate its infinitesimal symmetry. These Lie vectors are considered as follows:

$$
\begin{aligned}
& X_{1}=\frac{\partial}{\partial x}, \\
& X_{2}=\alpha x \frac{\partial}{\partial x}+2 t \frac{\partial}{\partial t}-\alpha u \frac{\partial}{\partial u} .
\end{aligned}
$$

Case 1. For (19), we have

$$
\frac{d x}{1}=\frac{d t}{0}=\frac{d u}{0}
$$

Solving this equation, $u=f(t)$. Putting $u=f(t)$ into (1), we get

$$
D_{t}^{\alpha} f(t)=0
$$

where $u=a_{1} t^{\alpha-1}$.

Case 2. For $X_{2}$ in (20), we have

$$
\frac{d x}{\alpha x}=\frac{d t}{2 t}=-\frac{d u}{\alpha u}
$$

This is the characteristic equation. By solving it, the resulting similarity variable in the form

$$
\begin{aligned}
& z_{1}=x t^{-\alpha / 2}, \\
& z_{2}=u t^{-\alpha / 2} .
\end{aligned}
$$

The variables transformation is as follows:

$$
u=t^{-\alpha / 2} f(\xi), \quad \xi=x t^{-\alpha / 2}
$$

where $f(\xi)$ is a function in one variable $\xi$. We use (25) to transform (2) into a fractional ODE.
Theorem 5. Transformation (25) reduces (2) to the nonlinear FODE as follows:

$$
\left(P_{3 / \alpha}^{1-3 \alpha / 2, \alpha} f\right)(\xi)+h f_{\xi \xi}+f f_{\xi}=0
$$

utilizing the Erdelyi-Kober (EK) fractional derivative operator [20]:

$$
\begin{aligned}
& \left(P_{\beta}^{\xi^{2}, \alpha} f\right)(\xi) \\
& \quad=\prod_{j=0}^{n-1}\left(\xi^{2}+j-\frac{1}{\beta} \frac{d}{d \xi}\right)\left(K_{\beta}^{\xi^{2}+\alpha, n-\alpha} f\right)(\xi),
\end{aligned}
$$

where

$$
\begin{aligned}
& \left(K_{\beta}^{\xi^{2}, \alpha} f\right)(\xi) \\
& = \begin{cases}\frac{1}{\Gamma(\alpha)} \int_{1}^{\infty}(u-1)^{\alpha-1} u^{-\left(\xi^{2}+\alpha\right)} f\left(\xi u^{1 / \beta}\right) d u, & \alpha>0 \\
f(\xi), & \alpha=0\end{cases}
\end{aligned}
$$

and

$$
n= \begin{cases}{[\alpha]+1,} & \alpha \neq N \\ \alpha, & \alpha \in N\end{cases}
$$

Proof. Utilizing the definition of the RL fractional derivative in (25), we get

$$
\begin{aligned}
& \frac{\partial^{\alpha} u}{\partial t^{\alpha}}=\frac{\partial^{n}}{\partial t^{n}}\left[\frac{1}{\Gamma(n-\alpha)}\right. \\
& \left.\cdot \int_{1}^{t}(t-s)^{n-\alpha-1} s^{-\alpha / 2} f\left(x s^{-(\alpha / 2)}\right) d s\right], \\
& n-1<\alpha<1, n=1,2, \ldots
\end{aligned}
$$

Assume that $v=t / s, d s=-\left(t / v^{2}\right) d v$. Thus, (30) becomes

$$
\begin{aligned}
& \frac{\partial^{\alpha} u}{\partial t^{\alpha}}=\frac{\partial^{n}}{\partial t^{n}}\left[t^{n-3 \alpha / 2} \frac{1}{\Gamma(n-\alpha)}\right. \\
& \left.\quad \int_{0}^{\infty}(v-1)^{n-\alpha-1} v^{-(n+1-3 \alpha / 2)} f\left(\xi v^{(\alpha / 2)}\right) d v\right]
\end{aligned}
$$


Applying the EK fractional integral operator (28) in (31), we have

$$
\frac{\partial^{\alpha} u}{\partial t^{\alpha}}=\frac{\partial^{n}}{\partial t^{n}}\left[t^{n-3 \alpha / 2}\left(K_{2 / \alpha}^{1-\alpha / 2, n-\alpha} f\right)(\xi)\right]
$$

For simplicity, we consider $=x t^{-\alpha / 2}, \phi \in(0, \infty)$. We thus find that

$$
t \frac{\partial}{\partial t} \phi(\xi)=t x\left(-\frac{\alpha}{2}\right) t^{-\alpha / 2-1} \phi(\xi)=-\frac{\alpha}{2} \xi \frac{\partial}{\partial \xi} \phi(\xi)
$$

Hence, we have

$$
\begin{aligned}
& \frac{\partial^{n}}{\partial t^{n}}\left[t^{n-3 \alpha / 2}\left(K_{2 / \alpha}^{1-\alpha / 2, n-\alpha} f\right)(\xi)\right]=\frac{\partial^{n-1}}{\partial t^{n-1}}\left[\frac{\partial}{\partial t}\right. \\
& \left.\cdot t^{n-3 \alpha / 2}\left(K_{2 / \alpha}^{1-\alpha / 2, n-\alpha} f\right)(\xi)\right] \\
& =\frac{\partial^{n-1}}{\partial t^{n-1}}\left[t^{n-3 \alpha / 2-1}\left(n-\frac{3 \alpha}{2}-\frac{\alpha}{2} \xi \frac{\partial}{\partial \xi}\right)\right. \\
& \left.\cdot\left(K_{2 / \alpha}^{1-\alpha / 2, n-\alpha} f\right)(\xi)\right] .
\end{aligned}
$$

Repeating $n-1$ times, we have

$$
\begin{aligned}
& \frac{\partial^{n}}{\partial t^{n}}\left[t^{n-3 \alpha / 2}\left(K_{2 / \alpha}^{1-\alpha / 2, n-\alpha} f\right)(\xi)\right]=\frac{\partial^{n-1}}{\partial t^{n-1}}\left[\frac{\partial}{\partial t}\right. \\
& \left.\cdot t^{n-3 \alpha / 2}\left(K_{2 / \alpha}^{1-\alpha / 2, n-\alpha} f\right)(\xi)\right]=\frac{\partial^{n-1}}{\partial t^{n-1}}\left[t^{n-3 \alpha / 2-1}(n\right. \\
& \left.\left.-\frac{3 \alpha}{2}-\frac{\alpha}{2} \xi \frac{\partial}{\partial \xi}\right)\left(K_{2 / \alpha}^{1-\alpha / 2, n-\alpha} f\right)(\xi)\right] \\
& =t^{-\alpha / 2} \prod_{j=0}^{n-1}\left[\left(1-\frac{3 \alpha}{2}+j-\frac{\alpha}{2} \xi \frac{\partial}{\partial \xi}\right)\left(K_{2 / \alpha}^{1-\alpha / 2, n-\alpha} f\right)\right.
\end{aligned}
$$

Applying the EK fractional differential operator (27) in (35), we get

$$
\begin{gathered}
\frac{\partial^{n}}{\partial t^{n}}\left[\left(t^{n-\alpha / 3}\left(K_{2 / \alpha}^{1-\alpha / 2, n-\alpha} f\right)(\xi)\right)\right] \\
=t^{-\alpha / 2}\left(P_{2 / \alpha}^{1-3 \alpha / 2, \alpha} f\right)(\xi)
\end{gathered}
$$

Substituting (36) into (32), we get

$$
\frac{\partial^{\alpha} u}{\partial t^{\alpha}}=t^{-\alpha / 2}\left(P_{2 / \alpha}^{1-3 \alpha / 2, \alpha} f\right)(\xi)
$$

Thus, (2) is reduced to a fractional-order ODE as follows:

$$
\left(P_{2 / \alpha}^{1-3 \alpha / 2, \alpha} f\right)(\xi)+f f_{\xi}+h f_{\xi \xi}=0
$$

\section{The Explicit Solution for the Time-Fractional Benjamin-Ono Equation by Using PSM}

The analytic solutions via PSM [26] are demonstrated. We assume that

$$
f(\xi)=\sum_{n=0}^{\infty} a_{n} \phi(\xi)^{n}
$$

Differentiating (39) twice regarding $\xi$, we get

$$
f^{\prime}(\xi)=\sum_{n=0}^{\infty} n a_{n} \phi(\xi)^{n-1},
$$

and

$$
f^{\prime \prime}(\xi)=\sum_{n=0}^{\infty} n(n-1) a_{n} \phi(\xi)^{n-2}
$$

Substituting (39), (40), and (41) into (38), we have

$$
\begin{aligned}
& \sum_{n=0}^{\infty} \frac{\Gamma(2-3 \alpha / 2-n \alpha / 2)}{\Gamma(2-\alpha / 2-n \alpha / 2)} a_{n} \phi(\xi)^{n} \\
& \quad+\sum_{n=0}^{\infty} a_{n} \phi(\xi)^{n} \sum_{n=0}^{\infty}(n+1) a_{n+1} \phi(\xi)^{n} \\
& +h \sum_{n=0}^{\infty}(n+2)(n+1) a_{n+2} \phi(\xi)^{n}=0
\end{aligned}
$$

Comparing coefficients in (42) when $n=0$, we obtain

$$
a_{2}=\frac{-1}{2 h}\left(\frac{\Gamma(2-3 \alpha / 2)}{\Gamma(2-\alpha / 2)} a_{0}+a_{0} a_{1}\right)
$$


When $n \geq 1$, the recurrence relations between the series coefficients are

$$
\begin{aligned}
& a_{n+2}=\frac{-1}{2 h(n+2)(n+1)}\left(\frac{\Gamma(2-3 \alpha / 2-n \alpha / 2)}{\Gamma(2-\alpha / 2-n \alpha / 2)} a_{n}\right. \\
& \left.\quad+(n+1) a_{n} a_{n+1}\right)
\end{aligned}
$$

Using (44), the series solution for (39) can be represented by substituting (43) and (44) into (39):

$$
\begin{aligned}
& f(\xi)=a_{0}+a_{1} \xi+a_{2} \xi^{2}+\sum_{n=1}^{\infty} a_{n+2} \xi^{n+2}=a_{0}+a_{1} \xi \\
& -\frac{1}{2 h}\left(\frac{\Gamma(2-3 \alpha / 2)}{\Gamma(2-\alpha / 2)} a_{0}+a_{0} a_{1}\right) \xi^{2} \\
& \quad-\sum_{n=1}^{\infty} \frac{1}{2 h(n+2)(n+1)}\left(\frac{\Gamma(2-3 \alpha / 2-n \alpha / 2)}{\Gamma(2-\alpha / 2-n \alpha / 2)} a_{n}\right. \\
& \left.\quad+(n+1) a_{n} a_{n+1}\right) \xi^{n+2} .
\end{aligned}
$$

Upon substitution using similarity variables in (25), the following explicit solutions for (2) are

$$
\begin{aligned}
& u(x, t)=a_{0} t^{-\alpha / 2}+a_{1} x t^{-\alpha}-\frac{1}{2 h}\left(\frac{\Gamma(2-3 \alpha / 2)}{\Gamma(2-\alpha / 2)} a_{0}\right. \\
& \left.+a_{0} a_{1}\right) t^{-\alpha / 2}\left(x t^{-\alpha / 2}\right)^{2} \\
& -\sum_{n=1}^{\infty}\left\{\frac{1}{2 h(n+2)(n+1)} \frac{\Gamma(2-3 \alpha / 2-n \alpha / 2)}{\Gamma(2-\alpha / 2-n \alpha / 2)} a_{n}\right. \\
& \left.+\sum_{k=0}^{n} \sum_{j=0}^{k}\left(a_{j}+a_{k-j}\right) a_{n-k}(n+1)\right\}(x)^{n+2} \\
& \cdot t^{-\alpha(2 n+\alpha \beta) / 2 \beta} .
\end{aligned}
$$

\section{Convergence Analysis}

To satisfy the convergence test, there are many kinds of tests as the ratio, the comparison, and the quotient tests. The convergence of the solution equation (46) will be presented as follows. We revamp (46) as follows:

$$
\begin{aligned}
&\left|a_{n+2}\right| \leq\left(\frac{|\Gamma(2-3 \alpha / 2-n \alpha / 2)|}{|\Gamma(2-\alpha / 2-n \alpha / 2)|}\left|a_{n}\right|\right. \\
&\left.-\sum_{k=0}^{n} \sum_{j=0}^{k}\left|a_{j}\right|\left|a_{k-j}\right|\left|a_{n-k}\right|-\left|a_{n}\right|\right)
\end{aligned}
$$

Equation (47), utilizing the Gamma function, shows that $|\Gamma(2-3 \alpha / 2-n \alpha / 2)| /|\Gamma(2-\alpha / 2-n \alpha / 2)|<1$ for arbitrary $n$ that

$$
\left|a_{n+2}\right| \leq M\left(\left|a_{n}\right|-\sum_{k=0}^{n} \sum_{j=0}^{k}\left|a_{j}\right|\left|a_{k-j}\right|\left|a_{n-k}\right|-\left|a_{n}\right|\right)
$$

where $M=\max \left\{\left(\left|c_{1}\right|,\left|c_{2}\right|\right)\right.$. We now assume another form of the PSM:

$$
B(\xi)=\sum_{n=0}^{\infty} c_{n} \xi^{n}
$$

By comparing the two series, we can observe that $\left|c_{n}\right| \leq a_{n}$, $n=0,1, \ldots$ Hence, the series $B(\xi)=\sum_{n=0}^{\infty} c_{n} \xi^{n}$ is the majorant series of (47). So, we find that

$$
\begin{aligned}
& B(\xi)=c_{0}+c_{1} \xi \\
& \quad+M\left(\sum_{n=0}^{\infty} c_{n} \xi^{2} B(\xi)+\sum_{n=0}^{\infty} \sum_{k=0}^{n} \sum_{j=0}^{k} c_{j} c_{k-j} c_{n-k}+\sum_{n=0}^{\infty} c_{n}\right) \\
& \quad \cdot \xi^{n+2}
\end{aligned}
$$

Consider an implicit functional system regarding $\xi$ as follows:

$$
\begin{aligned}
\beta(\xi, B) & \\
= & B-c_{0}-c_{1} \xi-c_{2} \xi^{2} \\
& \quad-M\left(\xi^{2} B(\xi)+2 B(\xi)^{2}+\left(\xi^{2}-c_{1} \xi-3 c_{0}\right) B\right)
\end{aligned}
$$

since $\beta$ is analytic in a neighborhood of $\left(0, c_{0}\right)$, where $\beta\left(0, c_{0}\right)=0$, and $(\partial / \partial B) \beta\left(0, c_{0}\right)=0$. Then, the series $B(\xi)=$ $\sum_{n=0}^{\infty} \xi^{n}$ is analytic around $\left(0, c_{0}\right)$ and this is verified utilizing [27] and the radius of convergence of this series belongs to a positive domain. This shows that (46) converges around $\left(0, c_{0}\right)$.

\section{Physical Performance of the Power Series Technique for Eqs. (46)}

To have expressed and convenient conception of the physical characteristic of the power series solution, the $3 \mathrm{D}$ plots for the explicit solution equations (46) is plotted in Figures 1-4 at $h=1$ by utilizing appropriate parameter forms. The spectacle vision of the real portion of (46) can be visible in the 3D plots proof in Figures 1, 2, 3, and 4, respectively. 


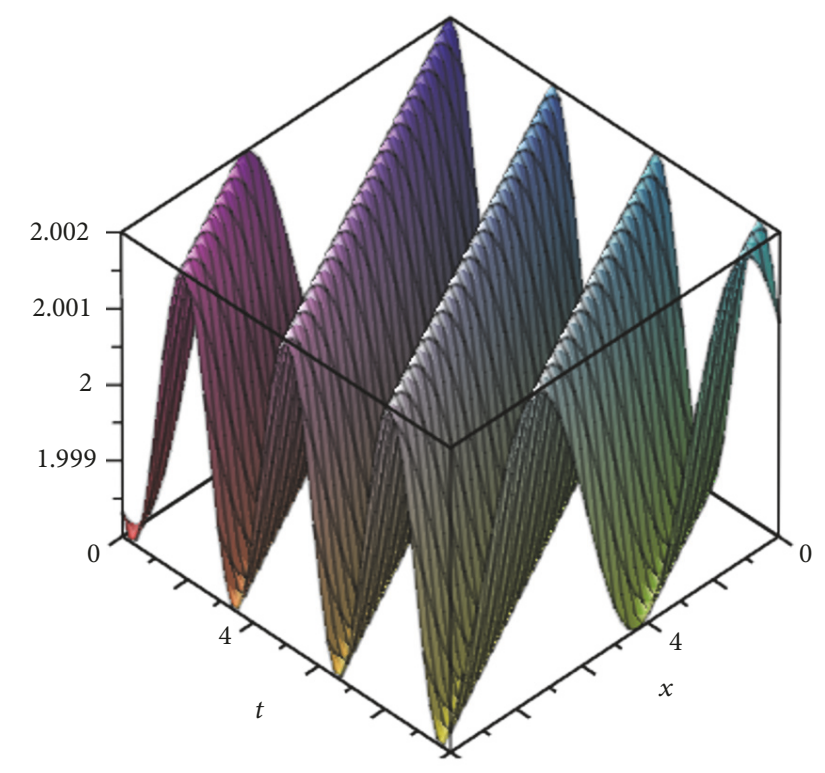

FIGURE 1: 3D plot of (46) with $a_{0}=1.9, a_{1}=1.7, a_{2}=0.77, \gamma=1$, $\alpha=0.8, \beta=2$.

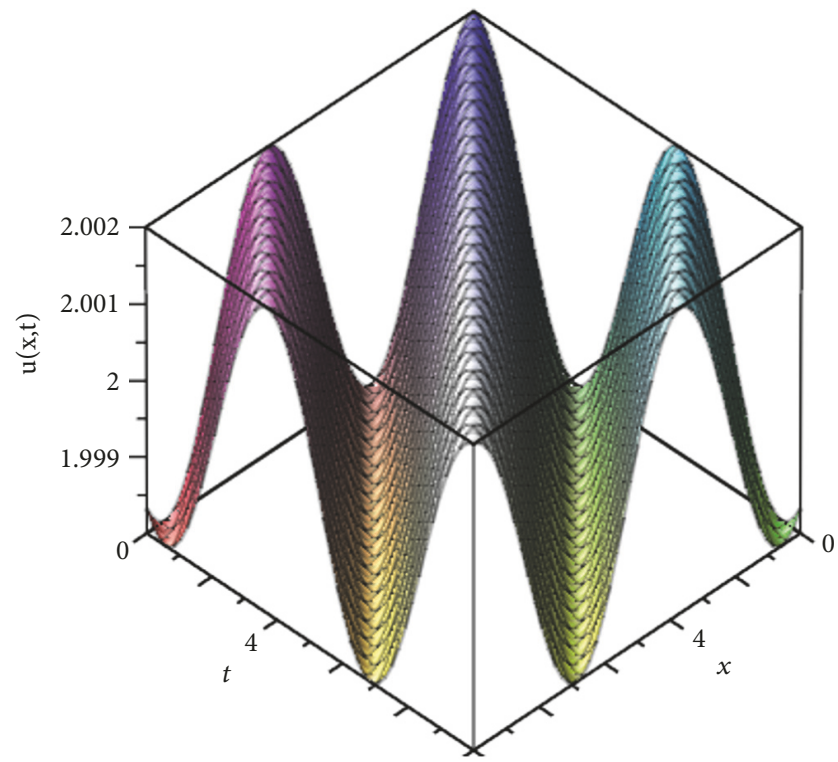

FIGURE 2: 3D plot of (46) with $a_{0}=3.9, a_{1}=1.7, a_{2}=0.87, \gamma=1$, $\alpha=0.6, \beta=1$.

\section{Conclusions}

Lie point symmetry properties of $(1+1)$-dimensional timefractional Benjamin-Ono equation have been considered with the Riemann-Liouville fractional derivative. These symmetries are used here to transform the FPDEs into NLFODEs. Closed-form solutions are determined by using PSM in the last division. The accuracy exhibits the assembly of the solution. Considerable frames for the acquired explicit solutions were approached.

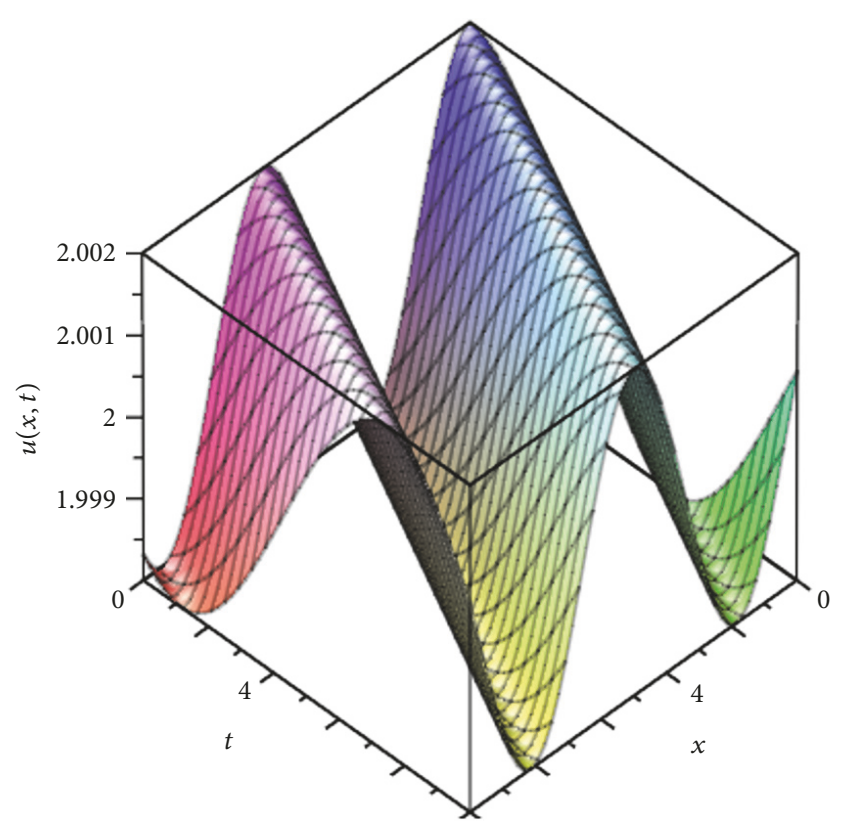

FIGURE 3: 3D plot of (46) with $a_{0}=1, a_{1}=1.1, a_{2}=0.57, \gamma=1$, $\alpha=0.5, \beta=0.7$.

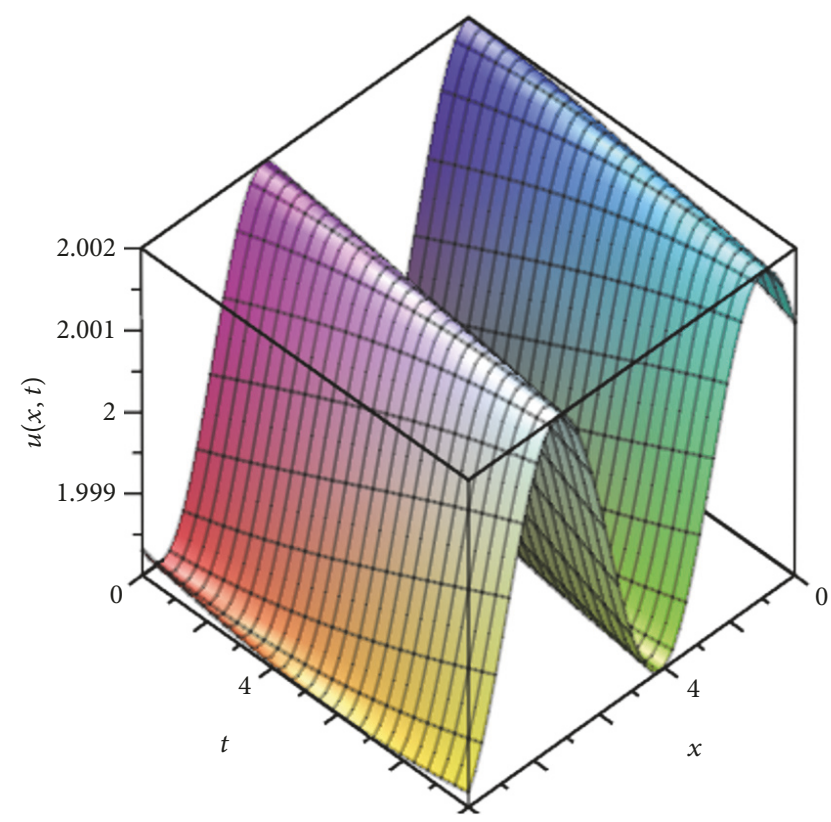

FIgURE 4: 3D plot of (46) with $a_{0}=1, a_{1}=1, a_{2}=0.4, \gamma=1$, $\alpha=0.3, \beta=0.4$.

\section{Data Availability}

The data used to support the findings of this study are available from the corresponding author upon request. 


\section{Conflicts of Interest}

The author declares that they have no conflicts of interest.

\section{References}

[1] M. Bruzon et al., "The symmetry reductions of a turbulence model," Journal of Physics A: Mathematical and General, vol. 34, no. 18, p. 3751, 2001.

[2] R. Sadat and M. Kassem, "Explicit Solutions for the $(2+1)$ dimensional jaulent-miodek equation using the integrating factors method in an unbounded domain," Mathematical \& Computational Applications, vol. 23, no. 1, p. 15, 2018.

[3] A. Paliathanasis and M. Tsamparlis, "Lie symmetries for systems of evolution equations," Journal of Geometry and Physics, vol. 124, pp. 165-169, 2018.

[4] Y. Y. Zhang, X. Q. Liu, and G. W. Wang, "Symmetry reductions and exact solutions of the $(2+1)$-dimensional Jaulent-Miodek equation," Applied Mathematics and Computation, vol. 219, no. 3, pp. 911-916, 2012.

[5] M. Mirzazadeh and M. Eslami, "Exact solutions of the Kudryashov-Sinelshchikov equation and nonlinear telegraph equation via the first integral method," Nonlinear Analysis: Modelling and Control, vol. 17, no. 4, pp. 481-488, 2012.

[6] M. Mirzazadeh, M. Eslami, A. H. Bhrawy, B. Ahmed, and A. Biswas, "Solitons and other solutions to complex-valued KleinGordon equation in $\Phi-4$ field theory," Applied Mathematics \& Information Sciences, vol. 9, no. 6, pp. 2793-2801, 2015.

[7] A. Nazarzadeh, M. Eslami, and M. Mirzazadeh, "Exact solutions of some nonlinear partial differential equations using functional variable method," Pramana-Journal of Physics, vol. 81, no. 2, pp. 225-236, 2013.

[8] M. Eslami, A. Neyrame, and M. Ebrahimi, "Explicit solutions of nonlinear (2+1)-dimensional dispersive long wave equation," Journal of King Saud University - Science, vol. 24, no. 1, pp. 69-71, 2012.

[9] M. Mirzazadeh, M. Eslami, and A. H. Arnous, "Dark optical solitons of Biswas-Milovic equation with dual-power law nonlinearity," The European Physical Journal Plus, vol. 130, 4, no. 1, 2015.

[10] E. Aksoy, M. Kaplan, and A. Bekir, "Exponential rational function method for space-time fractional differential equations," Waves in random and complex media : propagation, scattering and imaging., vol. 26, no. 2, pp. 142-151, 2016.

[11] A. Bekir and A. C. Cevikel, "New exact travelling wave solutions of nonlinear physical models," Chaos, Solitons and Fractals, vol. 41, no. 4, pp. 1733-1739, 2009.

[12] M. Eslami, M. Mirzazadeh, B. Fathi Vajargah, and A. Biswas, "Optical solitons for the resonant nonlinear Schrödinger's equation with time-dependent coefficients by the first integral method," Optik - International Journal for Light and Electron Optics, vol. 125, no. 13, pp. 3107-3116, 2014.

[13] M. F. El-Sabbagh, R. Zait, and R. M. Abdelazeem, "New exact solutions of some nonlinear partial differential equations via the improved exp-function method," IJRRAS, vol. 18, no. 2, pp. 132144, 2014.

[14] Y. Gurefe, E. Misirli, A. Sonmezoglu, and M. Ekici, "Extended trial equation method to generalized nonlinear partial differential equations," Applied Mathematics and Computation, vol. 219, no. 10, pp. 5253-5260, 2013.
[15] A. H. Khater, M. H. Moussa, and S. F. Abdul-Aziz, "Invariant variational principles and conservation laws for some nonlinear partial differential equations with constant coefficients - I," Chaos, Solitons and Fractals, vol. 14, no. 9, pp. 1389-1401, 2002.

[16] Q. Feng and F. Meng, “Traveling wave solutions for fractional partial differential equations arising in mathematical physics by an improved fractional Jacobi elliptic equation method," Mathematical Methods in the Applied Sciences, vol. 40, no. 10, pp. 3676-3686, 2017.

[17] R. K. Gazizov, A. A. Kasatkin, and S. Y. Lukashchuk, "Continuous transformation groups of fractional differential equations," Vestnik Usatu, vol. 9, no. 3, p. 21, 2007.

[18] S. Zhang and H. Q. Zhang, "Fractional sub-equation method and its applications to nonlinear fractional PDEs," Physics Letters A, vol. 375, no. 7, pp. 1069-1073, 2011.

[19] B. Zheng, "A new fractional Jacobi elliptic equation method for solving fractional partial differential equations," Advances in Difference Equations, vol. 2014, no. 1, p. 228, 2014.

[20] A. Neyrame, A. Roozi, S. S. Hosseini, and S. M. Shafiof, "Exact travelling wave solutions for some nonlinear partial differential equations," Journal of King Saud University - Science, vol. 22, no. 4, pp. 275-278, 2010.

[21] K. B. Oldham and J. Spanier, The Fractional Calculus Theory and Applications of Differentiation and Integration to Arbitrary Order, vol. 111, Academic Press, New York, NY, USA, London, UK, 1974

[22] B. Ahmad, S. K. Ntouyas, and A. Alsaedi, "On a coupled system of fractional differential equations with coupled nonlocal and integral boundary conditions," Chaos, Solitons \& Fractals, vol. 83, pp. 234-241, 2016.

[23] V. S. Kiryakova, Generalized Fractional Calculus and Applications, CRC press, Botan Roca, Fl, USA, 1993.

[24] G.-W. Wang, X.-Q. Liu, and Y.-Y. Zhang, "Lie symmetry analysis to the time fractional generalized fifth-order KdV equation," Communications in Nonlinear Science and Numerical Simulation, vol. 18, no. 9, pp. 2321-2326, 2013.

[25] S. S. Ray and S. Sahoo, "Invariant analysis and conservation laws of $(2+1)$ dimensional time-fractional ZKBBM equation in gravity water waves," Computers \& Mathematics with Applications, 2017.

[26] G.-W. Wang and T.-Z. Xu, "Invariant analysis and exact solutions of nonlinear time fractional Sharma-Tasso-Olver equation by Lie group analysis," Nonlinear Dynamics, vol. 76, no. 1, pp. 571-580, 2014.

[27] M. Inc, A. Yusuf, A. I. Aliyu, and D. Baleanu, "Lie symmetry analysis, explicit solutions and conservation laws for the spacetime fractional nonlinear evolution equations," Physica A: Statistical Mechanics and its Applications, vol. 496, pp. 371-383, 2018. 


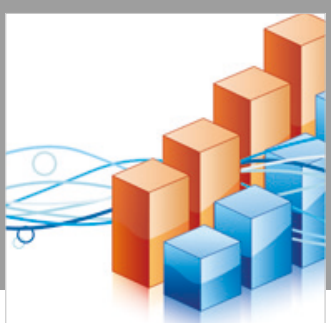

Advances in

Operations Research

\section{-n-m}
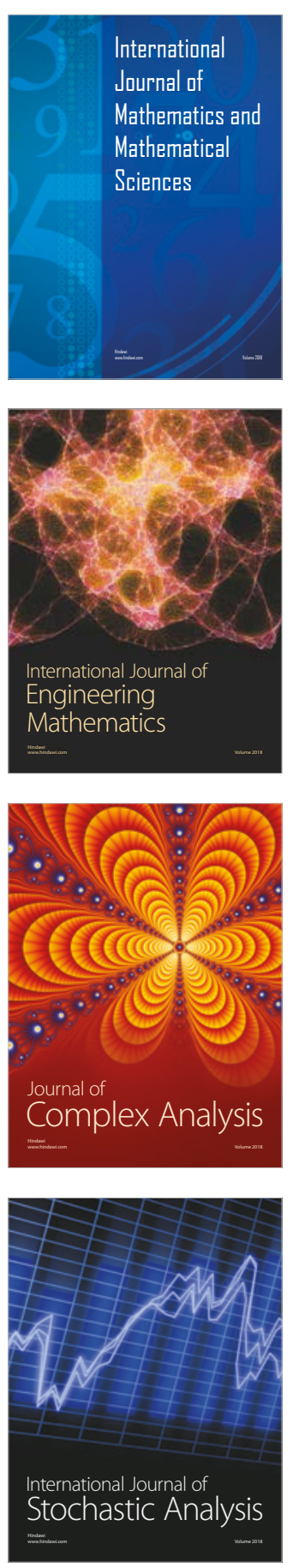
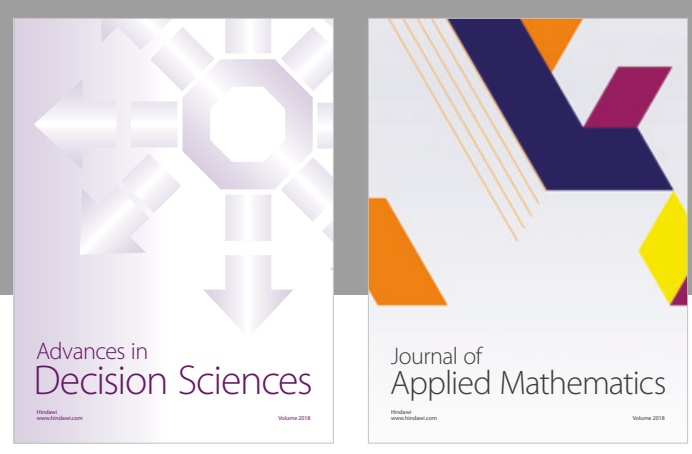

Journal of

Applied Mathematics
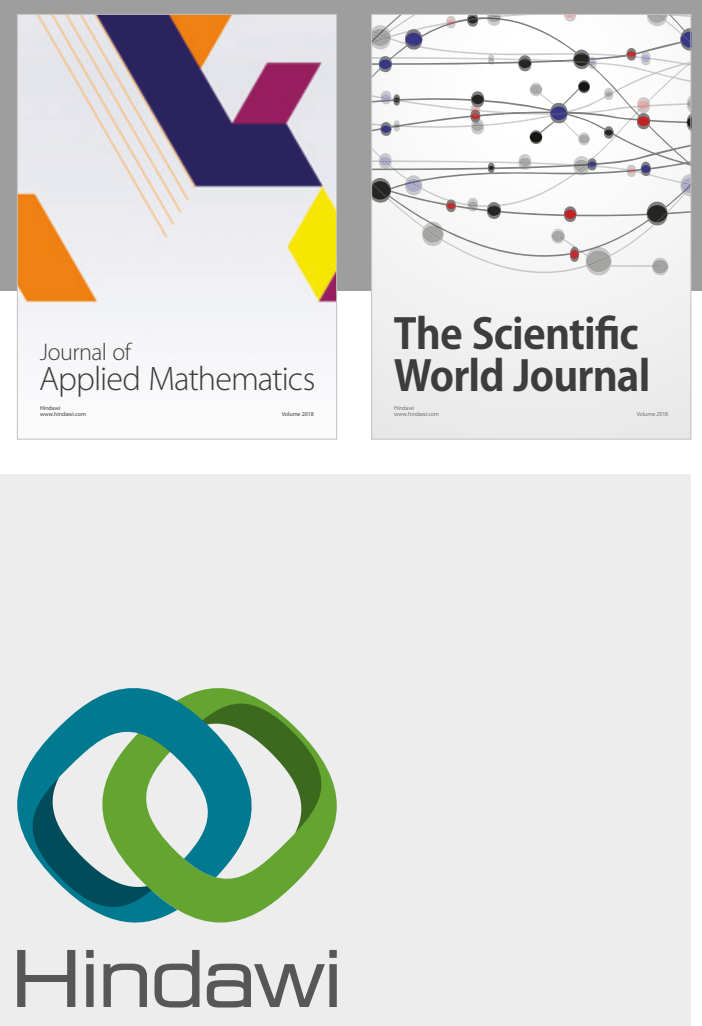

Submit your manuscripts at

www.hindawi.com

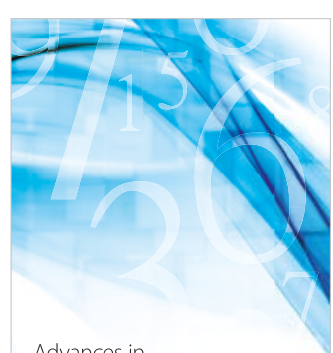

Advances in
Numerical Analysis
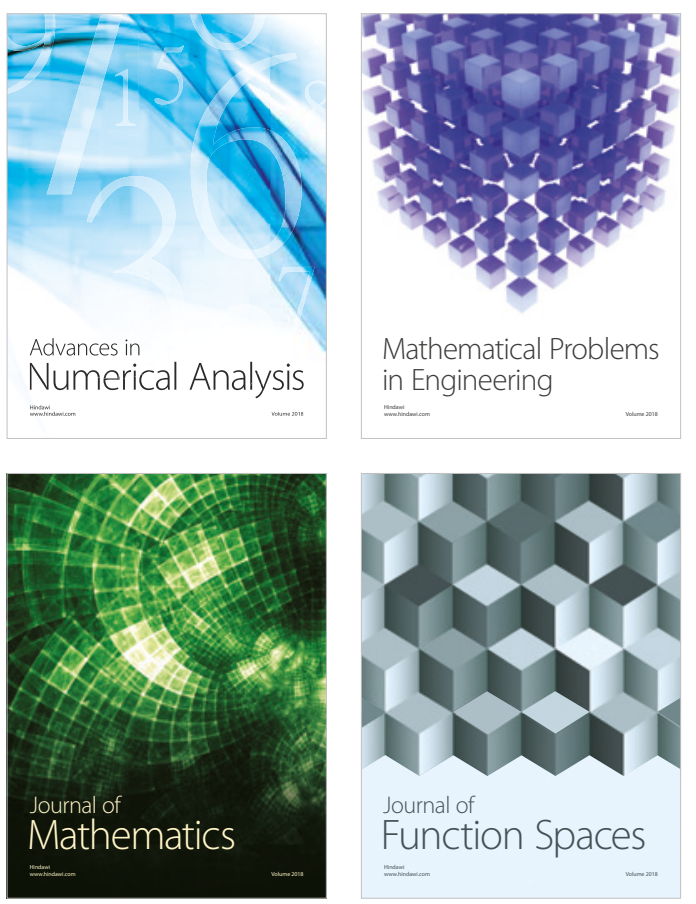

Mathematical Problems in Engineering

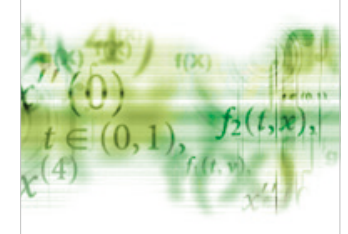

International Journal of

Differential Equations

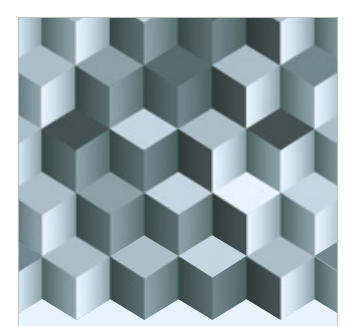

Journal of

Function Spaces

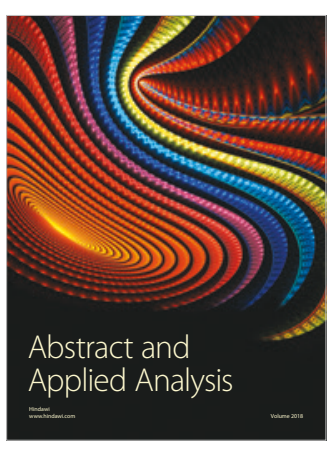

The Scientific

World Journal

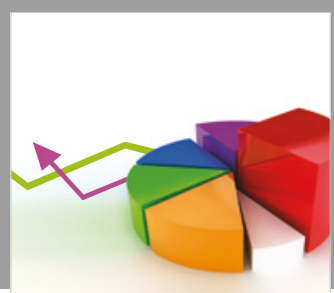

Journal of

Probability and Statistics
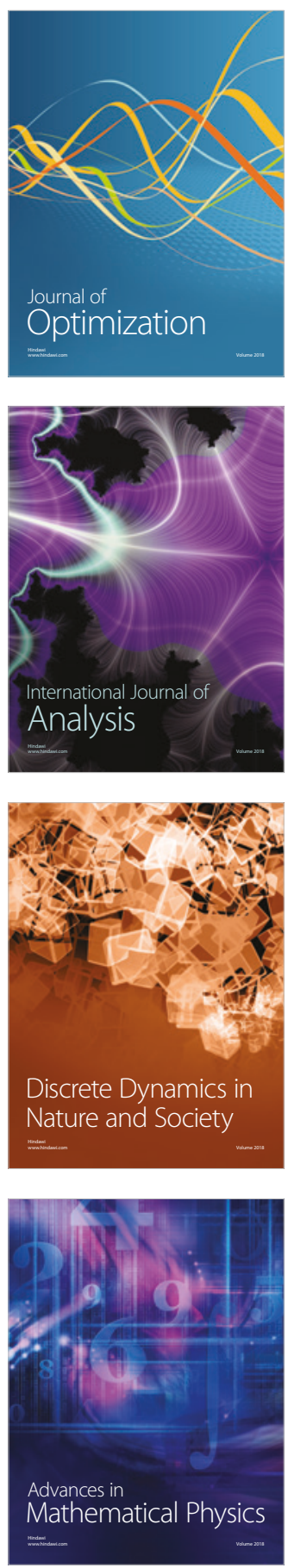